\title{
Comparison of organic diode structures regarding high-frequency rectification behavior in radio-frequency identification tags
}

\author{
Soeren Steudel ${ }^{\text {a),b) }}$ Stijn De Vusser, ${ }^{\text {b) }}$ Kris Myny, Martijn Lenes, ${ }^{\text {c) }}$ Jan Genoe, and \\ Paul Heremans ${ }^{b)}$ \\ IMEC, Polymer and Molecular Electronics, Kapeldreef 75, 3001 Leuven, Belgium
}

(Received 6 December 2005; accepted 13 March 2006; published online 15 June 2006)

\begin{abstract}
In this article, we compare the direct current (dc) and high-frequency performance of two different organic diode structures, a vertical diode and an organic field effect transistor (OTFT) with shorted drain-gate contact, regarding their application in a rectifying circuit. For this purpose, we fabricated both diode structures using the organic semiconductor pentacene. dc measurements were performed showing a space-charge-limited current mobility of more than $0.1 \mathrm{~cm}^{2} / \mathrm{V} \mathrm{s}$ for the vertical diode and a field effect mobility of $0.8 \mathrm{~cm}^{2} / \mathrm{V}$ s for the OTFT with shorted source-drain. High-frequency measurements of those diode structures in a rectifier configuration show that both types of diodes are able to follow the base-carrier frequency of $13.56 \mathrm{MHz}$ which is essential for viable radio-frequency-identification (rf-ID) tags. Based on those results we evaluate the performance limits and advantages of each diode configuration regarding their application in an organic rf-ID tag. (C) 2006 American Institute of Physics. [DOI: 10.1063/1.2202243]
\end{abstract}

\section{INTRODUCTION}

In the last decade, the performance of organic thin film transistor (OTFT) improved rapidly. Two potential applications motivate the work on OTFTs. On one hand, there is a great interest in using OTFTs in active matrix pixel drivers for displays, possibly on flexible substrates. Another focus of OTFT research is low-cost integrated circuits, in particular, passive radio-frequency-identification (rf-ID) tags (Fig. 1.). Circuits such as oscillators, shift registers, and even full tags working at $125 \mathrm{kHz}$ have already been demonstrated. ${ }^{1-4}$ However, in order to be a competitive and viable alternative, the organic rf-ID tag would need to be able to reach the already commercially used base-carrier frequency of 13.56 MHz, ${ }^{5}$ which is challenging because of the intrinsically low charge carrier mobility in organic semiconductors. The rectification stage in its simplest realization consists of a diode in series with a load capacitance to which the other stages of the circuit are connected in parallel as a load. The rectifying stage in a $13.56 \mathrm{MHz}$ rf-ID tag is, by far, the most critical part regarding speed, because the rectifying element needs to be able to charge the capacitance of the rectifier in only a fraction of the carrier frequency period. The circuitry of the logic operates at a much lower frequency, which depends on the required data rate and is commonly in the range of several hundred kilohertz. ${ }^{5}$ The gate of the load modulator transistor is also driven at this lower data rate, whereas the source-drain voltage follows the base-carrier frequency. The specifications for the load modulator transistor are less stringent than for the rectifying element, due to the much larger

\footnotetext{
${ }^{\text {a) }}$ Electronic mail: ssteudel@imec.be

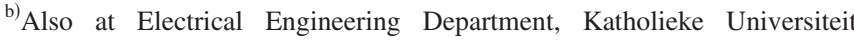
Leuven.

${ }^{c)}$ Also at Physics Department, University of Groningen.
}

voltage over the element and the availability of the full period to modulate. Moreover, only a partial modulation of the antenna load current is already sufficient.

It has already been suggested to use an OTFT with shorted gate-drain as a diode, ${ }^{6}$ which has the advantage of easy integration in the same process steps as the logic circuit. Furthermore the field effect mobility shown for OTFTs is generally orders of magnitude higher than the space-chargelimited current (SCLC) mobility relevant for vertical diodes, which is due to the higher charge density achieved in the channel of transistors compared to diode structures. ${ }^{7}$ One obvious drawback of a transistor-structure regarding speed is the long channel length compared to the thin organic semiconductor layer required for Schottky diodes. This drawback may exacerbate in the future as the process of fabricating transistors shifts away from laboratory-scale high-resolution photolithography processes, that allow channel lengths down to $1 \mu \mathrm{m}$, to high-volume production utilizing cheap printing processes with lower resolution $(>10 \mu \mathrm{m})$. Recently, Cantatore et $a l^{8}$ have shown that in principle it is possible to rectify an incoming ac signal at $13.56 \mathrm{MHz}$ using a transistor-based diode even though the rectified dc voltage was only $10 \mathrm{~V}$ using an ac amplitude of $80 \mathrm{~V}$.

An alternative implementation lays in the realization of a

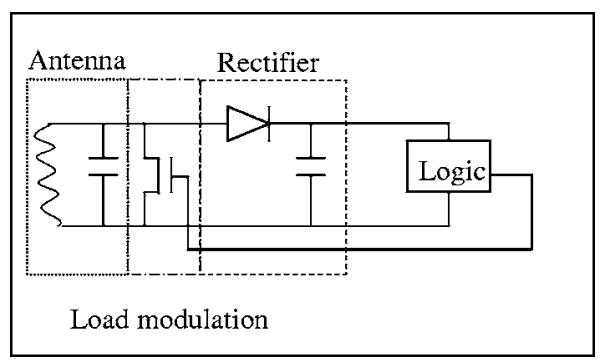

FIG. 1. Basic schematic diagram of a radio-frequency identification (rf-ID) tag. 
(a)

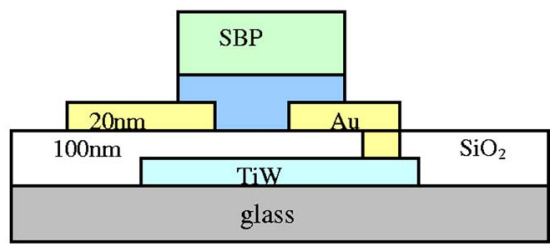

(b)

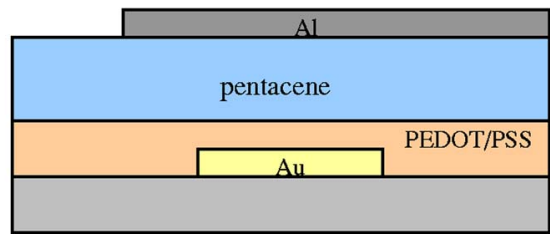

FIG. 2. (Color online) Experimental diode structures. (a) Organic field effect transistor with shorted drain-gate and (b) vertical organic diode.

vertical organic diode. The rectification is obtained by the different injection barriers between the two different metals and the organic semiconductor. Regardless of the injection model applicable to metal-organic interfaces, a metal-organic contact with a sufficiently large injection barrier will be blocking. A contact with a small injection barrier can be Ohmic. There are numerous publications about organic light-emitting diodes (OLEDs) that are based on a metalorganic semiconductor structure. ${ }^{9,10}$ Metal-organic semiconductor diodes using poly(2-methoxy,5-(2'-ethyl-hexoxy)-1,4phenylenevinylene) (MEH-PPV) sandwiched between $\mathrm{Al}$ and $\mathrm{Cu} /$ poly (3,4-)ethylenedioxythiophene (PEDOT/PSS) with $\mu_{\mathrm{SCLC}} \sim 10^{-6} \mathrm{~cm}^{2} / \mathrm{V} \mathrm{s}$ have already been reported. ${ }^{11}$ The highest SCLC mobility values reported for symmetric $\mathrm{Au}$ contacts for organic thin films of [2,2]-paracyclophane in a coplanar structure $\left(\mu_{\mathrm{SCLC}} \sim 10^{-2} \mathrm{~cm}^{2} / \mathrm{V} \mathrm{s}\right)($ Ref. 12) and for doped zinc pthalocyanine $\left(\mu_{\mathrm{SCLC}} \sim 10^{-1} \mathrm{~cm}^{2} / \mathrm{V} \mathrm{s}\right)$ (Ref. 13) are still below the mobility values reported for OTFTs. Recently, we reported about a pentacene based diode with current densities up to $2000 \mathrm{~A} / \mathrm{cm}^{2}$ and a mobility of $\sim 0.15 \mathrm{~cm}^{2} / \mathrm{V} \mathrm{s}$, ${ }^{14}$ which can rectify an incoming ac signal of $18 \mathrm{~V}$ amplitude to a de signal of $11 \mathrm{~V}$ at $13.56 \mathrm{MHz}$ sufficient to drive a circuit.

\section{EXPERIMENT}

We have fabricated pentacene transistors on glass with $100 \mathrm{~nm}$ sputtered $\mathrm{SiO}_{2}$ as gate dielectric as described in detail elsewhere. ${ }^{15}$ The drain and gate contacts are shorted and transistors with different channel lengths and widths are available on the sample [Fig. 2(a)].

Vertical diodes of different sizes using highly purified, vacuum-deposited pentacene ${ }^{14}$ [Fig. 2(b)] have been fabricated too. Pentacene was chosen because it can be considered the base line material for maximum mobility in organic layers. The injection electrode is a lithographically patterned line of $20 \mathrm{~nm} \mathrm{Au}$ with and without $30 \mathrm{~nm}$ PEDOT/PSS as a hole-injection layer. The semiconducting layer is $300 \mathrm{~nm}$ of pentacene deposited at a temperature of $20^{\circ} \mathrm{C}$ with a deposition rate of $\sim 3 \AA / \mathrm{s}$. The top $\mathrm{Al}$ electrode is deposited through a shadow mask.

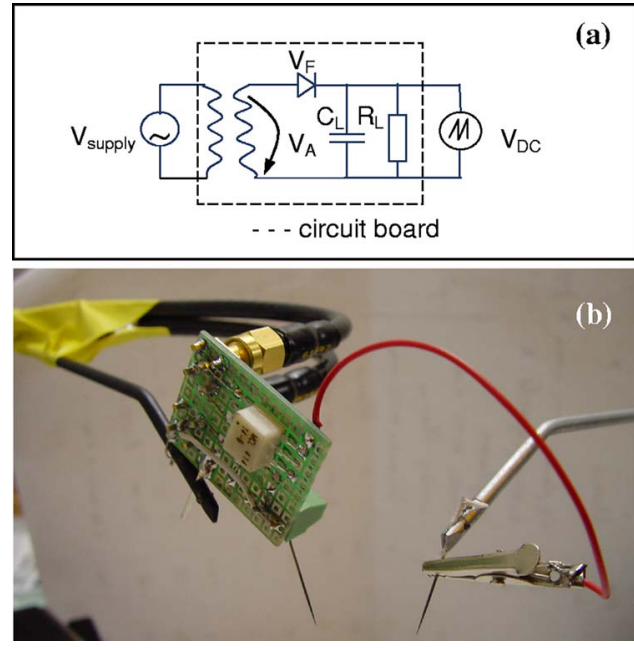

FIG. 3. (Color online) High-Frequency rectification measurement setup. (a) Schematic and (b) photograph.

The chosen geometries were designed for the two different devices to be able to operate in a similar voltage range, meaning that the structures sustain a forward bias of $>15 \mathrm{~V}$, a reverse bias of $>25 \mathrm{~V}$, and achieve a high current at a small capacitance. Those requirements are explained in more detail in the Discussion section.

We performed quasistatic current measurements on those devices as well as high-frequency measurement in a rectifier configuration. All measurements were done in a $\mathrm{N}_{2}$-purged glove box to prevent degradation. At frequencies over $1 \mathrm{MHz}$, the cable of the measurement setup distorts the measurement signal. Therefore we designed the measurement setup depicted in Fig. 3. The high-frequency input signal is decoupled from the output signal by a microtransformer which has the additional purpose to increase the amplitude of the ac signals because standard sinus generators such as the HP $8116 \mathrm{~A}$ do not supply voltage amplitude above $10 \mathrm{~V}$. The load capacitance $C_{\text {load, }}$, the load resistance $R_{\text {load, }}$, and the probe tips are attached to a minicircuit board that also contains the microtransformer to reduce the inductance of the connections. The measured signal is only the already rectified dc signal over the load resistor to which an oscilloscope with an input impedance of $1 \mathrm{M} \Omega$ is connected in parralel.

\section{RESULTS}

Figure 4(a) shows the transistor transfer curves $\left(I_{D^{-}} V_{G}\right)$ in saturation of transistors of our process. The transistors with a $W / L$ of $5000 / 10,2500 / 5$, and $1500 / 3$ have a mobility of $\mu \sim 0.8 \mathrm{~cm}^{2} / \mathrm{V} \mathrm{s}$ and a threshold voltage $V_{T} \sim 2.4 \mathrm{~V}$. Figure $4(\mathrm{~b})$ shows the $I-V$ characteristics of transistor with shorted gate-drain with various $W / L$. They exhibit a good rectifying behavior with an $I_{\mathrm{on}} / I_{\mathrm{off}}$ ratio of $\sim 100$. The relatively high off current is caused by the slightly positive threshold voltage.

The $I-V$ characteristics of the vertical diode structures are depicted in Fig. 5(a). The samples with a PEDOT/PSS injection layer reach a current density exceeding $2000 \mathrm{~A} / \mathrm{cm}^{2}$, roughly five times higher as compared to the UV-ozone cleaned sample. However, the reverse current is 

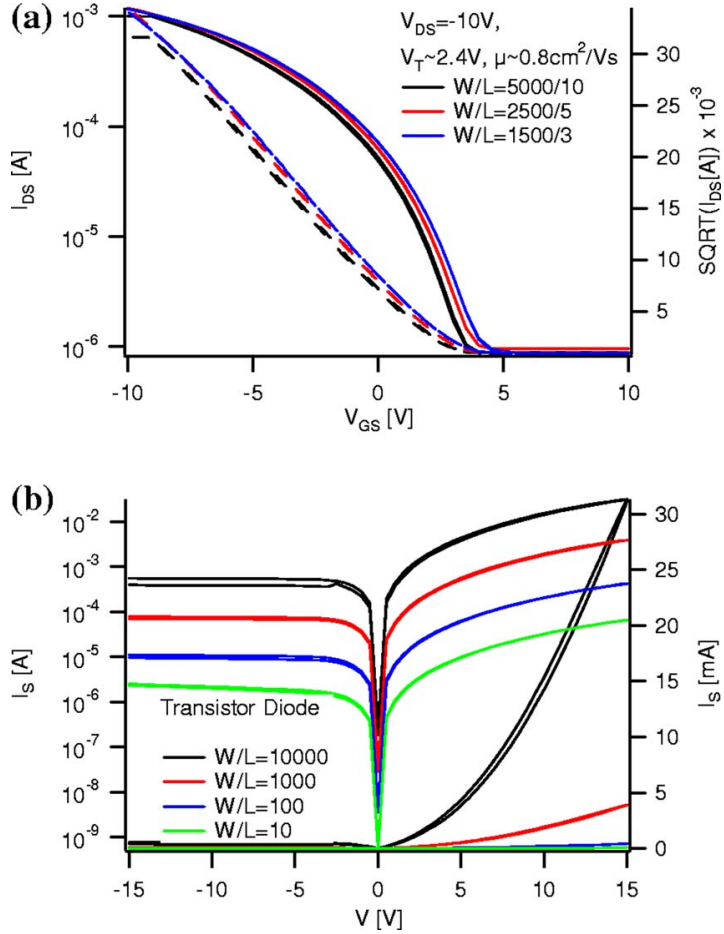

FIG. 4. (Color online) Transistor-based diode. (a) Transfer curve of transistor and (b) current-voltage characteristics.

significantly better in the diode without PEDOT/PSS. The high reverse current in the sample with PEDOT/PSS is mostly due to the lateral current between the probe needles. Separately fabricated samples with high-conductive PEDOT/ PSS showed a much higher reverse current compared to samples with low-conductive PEDOT/PSS. Scratching the pentacene and PEDOT/PSS around the diode area improved the off current. Patterning the organic semiconductor as well as the PEDOT/PSS might significantly improve the rectification. Measuring those samples in air decreased the maximum current by more than one order of magnitude, which can be linked to a reduced hole mobility due to humidity. ${ }^{16}$ On a $\mathrm{Si} / \mathrm{SiO}_{2}$ substrate the diode sustains a forward bias of $19 \mathrm{~V}$ and a reverse bias larger than $30 \mathrm{~V}$. Using a glass substrate the breakdown voltage under forward bias is reduced to $7 \mathrm{~V}$ which can be traced back to the low heat conductivity of the glass. ${ }^{14}$ This lower breakdown voltage on glass is only an issue for the dc measurement of the $I-V$ curve. Measuring under HF conditions (megahertz), the diode on glass can work in the same voltage range as a comparable diode on a $\mathrm{Si} / \mathrm{SiO}_{2}$ substrate.

From the double-logarithmic plot shown in Fig. 5(c), it can be seen that the current-voltage $(I-V)$ characteristics of the sample without PEDOT/PSS has a slope significantly larger than 2, which implies injection-limited charge transport. ${ }^{17}$ By contrast, the sample with PEDOT/PSS exhibits a slope of 2 over a voltage range of nearly one order of magnitude, which is an indication of trap-free (SCLC). Therefore it is possible to extract the hole mobility with help of Child's law ${ }^{17}$ out of the $I-V$ characteristics of the device with PEDOT/PSS. The thickness of the pentacene layer after deposition as measured with a profilometer is $300 \mathrm{~nm}$. However, it must be kept in mind that the pentacene layer is
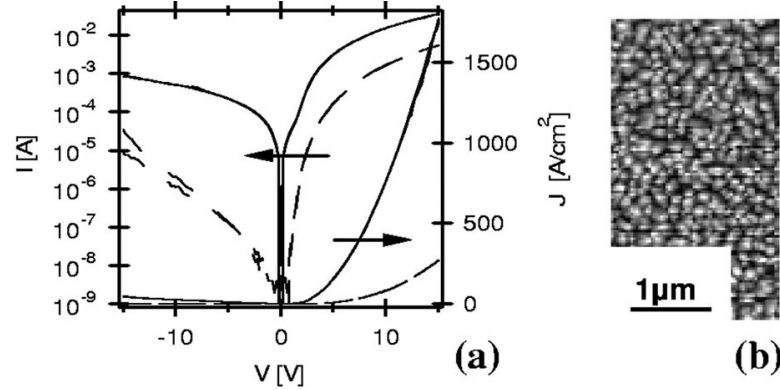

(b)
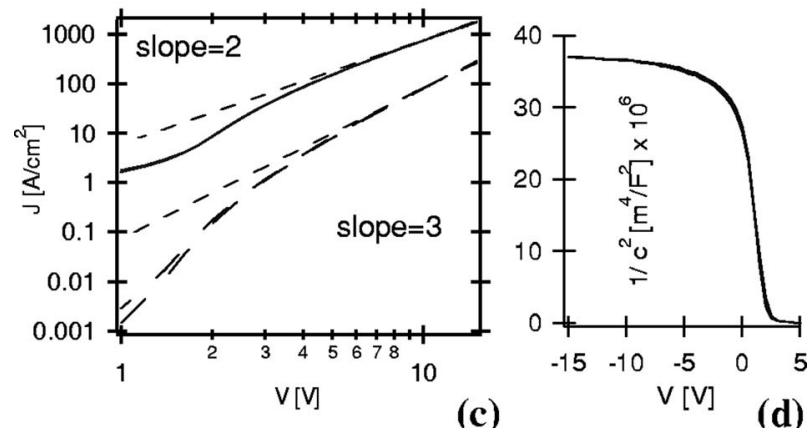

FIG. 5. Vertical pentacene diode. (a) Current-voltage characteristics $(A$ $\sim 170 \times 10 \mu \mathrm{m}^{2}$ ), (b) atomic force microscopic image of polycrystalline pentacene, (c) log-log plot of $I-V$ curve, and (d) capacitance-voltage plot.

polycrystalline as depicted in Fig. 5(b), and therefore the relevant thickness is the equivalent electrical thickness. We extracted this thickness of the polycrystalline pentacene layer in the device from high frequency $(f=100 \mathrm{kHz})$ capacitorvoltage measurements in reverse bias. This measurement results in a value of $\sim 160 \mathrm{~nm}$ [Fig. 5(d)]. Assuming this thickness value, Child's law applied to the $I-V$ characteristics of Fig. 5(c) allows to estimate a mobility of $0.15 \mathrm{~cm}^{2} / \mathrm{V} \mathrm{s}$. No significant difference of the value for the depletion capacitance has been observed between the samples with PEDOT/ PSS and without PEDOT/PSS.

The achieved SCLC mobility is extremely high. This can be traced back to two causes. On the one side, we used pentacene purified in forming gas ${ }^{14}$ and measured in a $\mathrm{N}_{2}$ atmosphere. The high semiconductor purity is attested by the temperature dependence of the mobility extracted from SCLC measurements (Fig. 6). Over a temperature range of

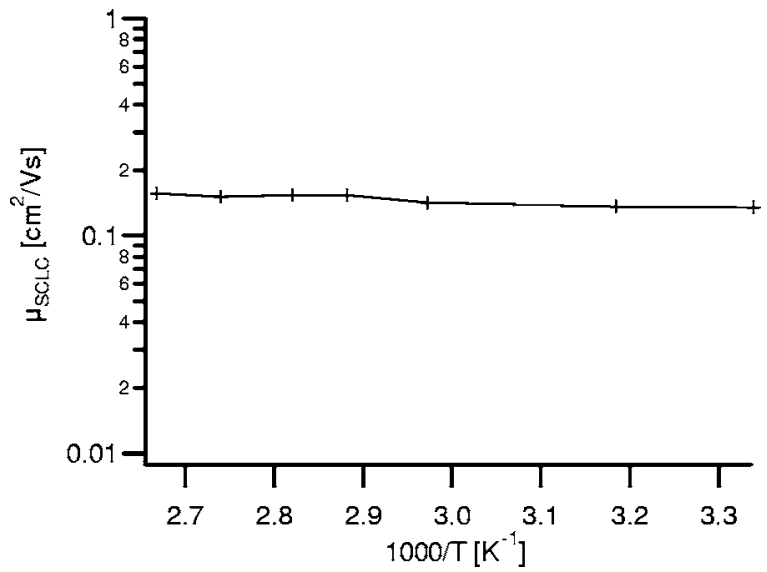

FIG. 6. Temperature dependence of the mobility in a vertical pentacene diode. 


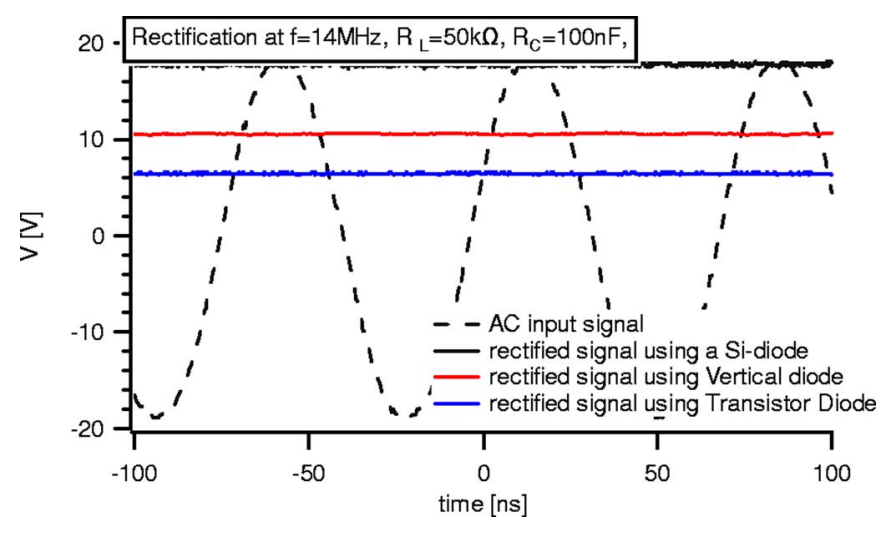

FIG. 7. (Color online) Rectifying signal using different diodes at $14 \mathrm{MHz}$ (transistor diode $\rightarrow W / L=3000 / 3$ and vertical diode $\rightarrow A \sim 170 \times 50 \mu \mathrm{m}^{2}$ ).

$80{ }^{\circ} \mathrm{C}$ the mobility increased by less than $10 \%$. This indicates very few or very shallow traps with low activation energy. ${ }^{18-20}$ The slope of the diode current in the Ohmic region of the diode does not increase significantly with temperature as would be expected, e.g., for injection by thermionic emission across a Schottky barrier. A more probable model takes into account the localized nature of the injected charge. $^{21}$

On the other hand the crystalline nature of the deposited pentacene film improves charge transport compared to that in a more amorphous film. It has been shown by Ma et al. ${ }^{22}$ that crystallization through annealing allows to increase the achievable current densities in a C60 film by more than two orders of magnitude.

After measuring the dc characteristics, the two types of diodes on glass were measured in a rectifier configuration using a load of $C_{\text {load }}=100 \mathrm{nF}, R_{\text {load }}=50 \mathrm{k} \Omega$, and an $18 \mathrm{~V}$ amplitude sinusoidal signal provided to the diode in series to the load resistor. In Fig. 7 the results for a frequency of $14 \mathrm{MHz}$ can be seen. As a reference value to demonstrate the reliability of the rectifier measurement setup, the rectified dc signal using a $\mathrm{Si}-\mathrm{pn}$ diode have been added.

\section{DISCUSSION}

Considering the rectifier stage in an organic rf-ID tag, it is necessary to outline the required performance. The possible working frequencies of rf-ID tags are given by the available base-carrier frequencies at $125 \mathrm{kHz}$ (LF), 13.56 MHz (HF), and $869 \mathrm{MHz}$ (Ref. 5) (UHF). The most commonly used base carrier frequency for Si-rf-ID tags is 13.56 MHz. To be compatible with installed readers, organic rf-ID tags should be able to work at $13.56 \mathrm{MHz}$ too. At $125 \mathrm{kHz}$ the reading distance of the rf-ID tag is very short and the demands on the antenna as well as on the size of the load capacitance are very strict. A second point to be considered is the supply voltage $V_{\text {supply }}$ needed to drive an organic circuit. Lower supply voltage results in a simpler power supply and a longer reading distance. There is no clear limit as to the maximum supply voltage, but a range of 5 to $10 \mathrm{~V}$ seems acceptable. A third boundary condition is the readout time of a rf-ID tag limited in practice to about $10 \mathrm{~ms}^{5}$

Assuming a standard rf-ID tag with 96 bit encoding, this translates in a stage delay for the shift register in the order of

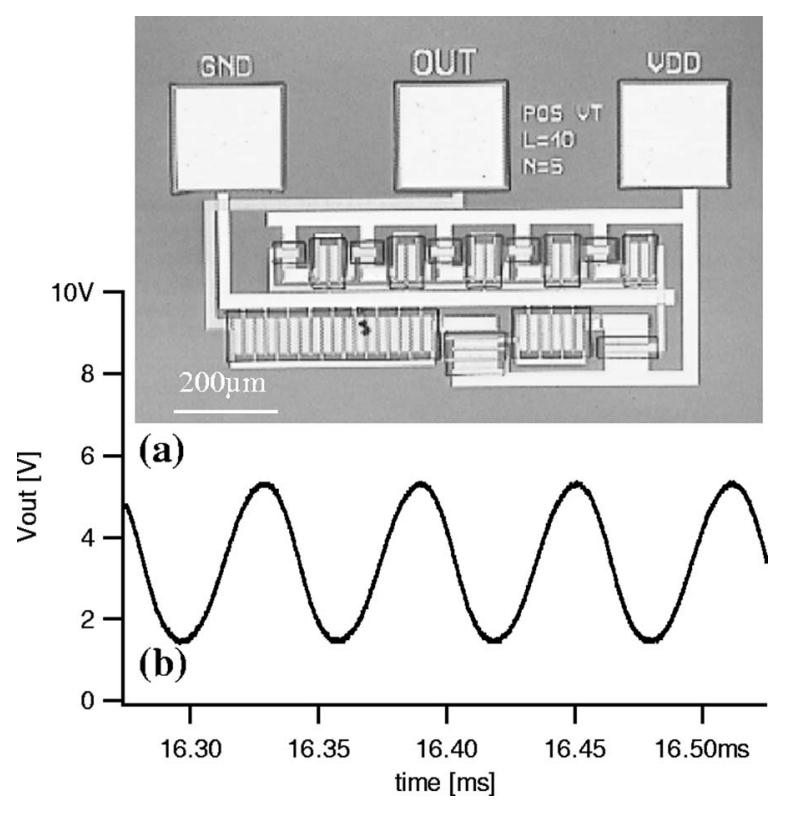

FIG. 8. Five-stage ring oscillator working at $10 \mathrm{~V}$ supply voltage.

$\sim 50 \mu$ s. Herby it needs to be held in mind, that the nonoverlapping clock signal for the shift register needs to be generated by an integrated oscillator which requires an at least one order of magnitude faster stage delay for the inverter in the oscillator as compared to the shift register, meaning a minimum stage delay of $5 \mu$ s. Taking into account necessary output buffer stages as well as anticollision procedures would require an even lower stage delay.

The lowest published supply voltage for driving an oscillator has been $1.5 \mathrm{~V}$ (Ref. 23) by using a gate-dielectric stack of self-assembly monolayer and thermal grown $\mathrm{Al}_{2} \mathrm{O}_{3}$, however, with a stage delay of $\sim 1 \mathrm{~ms}$. Circuits using organic or inorganic thin film dielectrics have recently been shown to operate with supply voltages as low as $8.5 \mathrm{~V} .{ }^{15}$ Based on the technology that we use for fabricating transistor-based diodes, we show in Fig. 8 a five-stage ring oscillator with output stage operating at $10 \mathrm{~V}$ supply voltage. The stage delay is $3.5 \mu \mathrm{s}$ at a current consumption of $\sim 5 \mu \mathrm{A}$. The oncurrent in a single inverter is $\sim 800 \mathrm{nA}$ and the off current is $\sim 40 \mathrm{nA}$. Based on those results we can make a rough estimate of the power consumption for a 96 bit rf-ID tag. Assuming 96 bits, the shift register consists of 192 inverters with half of the inverters in the on state and half in the off state. In addition, two clock signals need to be generated by an oscillator (e.g., 19 stage oscillator) with output stage and two NANDs. These elements will draw an estimated $100 \mu \mathrm{A}$ from the power supply.

Summarizing those requirements, the rectifier based on an organic diode needs to be able to deliver a supply voltage of $10 \mathrm{~V}$ and a current of $100 \mu \mathrm{A}$ from an incoming rf signal at $13.56 \mathrm{MHz}$.

To give an insight regarding the frequency limits of an organic rectifier we estimate the maximum frequency as a function of the voltage, mobility, dielectric thickness, and channel length for the transistor-based diode. Those calculations have already been shown elsewhere for the vertical diode structures. ${ }^{14}$ The analysis for an OTFT-based rectifier 
follows here. In our calculations we assume that (i) there is no leakage current through the diode in reverse bias, (ii) the time for charging the load capacitance during startup is not a limiting factor, and (iii) the area of the diode as well as of the load capacitor can be freely chosen without any concern regarding available substrate area.

Because a rectified dc voltage of $10 \mathrm{~V}$ is needed, calculations based on small signal parameters are not valid, but a large-signal analysis is required. The maximum theoretical frequency is determined by the speed with which the charges consumed by the load $\left(R_{L}\right)$ at a dc voltage $V_{\mathrm{dc}}$ during one frequency cycle $(\omega)$ can be recharged onto the load capacitance $\left(\mathrm{C}_{L}\right)$ by the current going through the organic diode during the fraction of the cycle that the diode is in forward bias,

$$
\frac{V_{\mathrm{dc}}}{R_{L}} \frac{2 \pi}{\omega}=\int_{t_{1}}^{t_{2}} I_{\text {diode_forward }} d t,
$$

where $t_{1}$ and $t_{2}$ delimit the time interval in which a forward bias is present over the diode.To get a stable dc voltage at the load, the discharging of the load capacitance $C_{L}$ over the load $R_{L}$ should be very small during one cycle, i.e., the following condition should be fulfilled:

$$
R_{L} C_{L} \gg \frac{2 \pi}{\omega} \Rightarrow R_{L} C_{L}=\frac{2 \pi}{\omega} N_{R C}
$$

where $N_{\mathrm{RC}}$ is a large numeric factor that determines the amplitude fluctuation on $V_{\mathrm{dc}}$ as a results of the $R C$ discharge in a cycle. In addition, the load capacitance needs to be very large compared to the diode capacitance. Indeed the ratio between load capacitance and diode capacitance defines the amplitude of the small signal ac voltage on top of the dc voltage,

$$
C_{L} \gg C_{D} \Rightarrow C_{L}=N_{C C} C_{D},
$$

where $N_{C C}$ is again a large numeric factor, that determines the amplitude fluctuation on $V_{\mathrm{dc}}$ as a result of the capacitive coupling of the ac supply voltage. As both $N_{R C}$ and $N_{C C}$ determine the amplitude fluctuation on $V_{\mathrm{dc}}$, we choose them to be equal: $N_{R C}=N_{C C}=N$. For a good rectifier, $N$ should be in the range from $\sim 100$ to $\sim 1000$. The diode capacitance corresponds to the geometrical capacitance of the transistorbased diode determined by the thickness of the dielectric $t_{\mathrm{ox}}$, the dielectric constant of the semiconductor $\varepsilon_{r}$ $\left(\varepsilon_{r \text {,sputtered, } \mathrm{SiO}_{2}} \sim 4.7\right)$, and the dielectric permittivity $\varepsilon_{0}$. The area of the transistor $A$ is itself the product of the width of the transistor channel $W$ and the length of the channel $L$ in addition to the overlap $L_{\text {overlap }}$ of the source/drain contact with the gate electrode.

$$
C_{D}=\frac{\varepsilon_{0} \varepsilon_{r} A}{t_{\mathrm{ox}}}=\frac{\varepsilon_{0} \varepsilon_{r} W\left(L+L_{\mathrm{overlapp}}\right)}{t_{\mathrm{ox}}} .
$$

The lower and upper bounds of integration in Eq. (1), $t_{1}$ and $t_{2}$, describe the time interval during which the supply voltage $\left(V_{A}\right)$ in forward direction is higher than the dc voltage $\left(V_{\mathrm{dc}}\right)$ of the load capacitance.

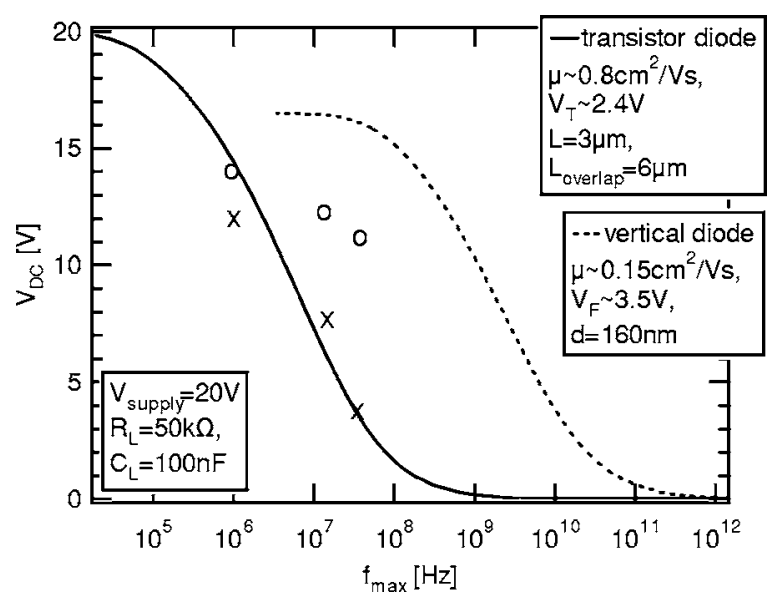

FIG. 9. Calculated (lines) and measured (crosses and circles) frequency dependences of the rectified dc voltage for a rectifier with a vertical organic diode and a transistor diode. The dotted line is calculated from (Ref. 14) based on a vertical diode structure and the following values are assumed: $\mu=0.15 \mathrm{~cm}^{2} / \mathrm{V} \mathrm{s}, L_{D}=160 \mathrm{~nm}, V_{F}=3.5 \mathrm{~V}$, and $V_{A}=20 \mathrm{~V}$; measured performance is indicated with open circles. The full line is calculated from Eq. (9) based on a transistor with shorted drain-gate and the following values are assumed: $\mu=0.8 \mathrm{~cm}^{2} / \mathrm{V} \mathrm{s}, \quad t_{\mathrm{ox}}=100 \mathrm{~nm}, \quad V_{T}=2.4 \mathrm{~V}, L=3 \mu \mathrm{m}, L_{\text {overlap }}$ $=6 \mu \mathrm{m}$, and $V_{A}=20 \mathrm{~V}$; measurements are shown as crosses.

$$
t_{1}=\sin ^{-1}\left(\frac{V_{\mathrm{dc}}}{V_{A}}\right) \frac{1}{\omega}, \quad t_{2}=\left[\pi-\sin ^{-1}\left(\frac{V_{\mathrm{dc}}}{V_{A}}\right)\right] \frac{1}{\omega} .
$$

The charging current $I_{\text {diode_forward }}$ can be described by the formula for a transistor in saturation. ${ }^{24}$ Hence

$$
I_{\text {diode_forward }}=\frac{\mu W \varepsilon_{0} \varepsilon_{r}\left(V_{\text {diode_forward }}-V_{T}\right)^{2}}{2 t_{\mathrm{ox}} L},
$$

where $I_{\text {diode_forward }}$ is defined as

$$
V_{\text {diode_forward }}=V_{A} \sin (\omega t)-V_{\text {dc }},
$$

and $W$ and $L$ are the width and the length of the transistor channel. Out of Eqs. (1)-(7) we get

$$
\begin{aligned}
\frac{\varepsilon_{0} \varepsilon_{r} A}{t_{\mathrm{ox}}} V_{\mathrm{dc}}= & \frac{\varepsilon_{0} \varepsilon_{r} \mu W}{2 t_{\mathrm{ox}} L} \int_{\sin ^{-1}\left(V_{\mathrm{dc}} / V_{A}\right) 1 / \omega}^{\left[\pi-\sin ^{-1}\left(V_{\mathrm{dc}} / V_{A}\right)\right] 1 / \omega} \\
& \times\left(V_{A} \sin (\omega t)-V_{\mathrm{dc}}-V_{T}\right)^{2} d t .
\end{aligned}
$$

Solving this equation yields the maximum frequency $f_{\text {max }}=\omega / 2 \pi$ as

$$
\begin{aligned}
f_{\max }= & \frac{\mu}{4 \pi L\left(L+L_{\text {overlapp }}\right) V_{\mathrm{dc}}} \\
& \times\left\{\left[-V_{A} \sqrt{1-\frac{V_{\mathrm{dc}}^{2}}{V_{A}^{2}}}\left(3 V_{\mathrm{dc}}+4 V_{T}\right)\right.\right. \\
& \left.+\left(V_{A}^{2}+2\left(V_{\mathrm{dc}}+V_{T}^{2}\right)\right] \arccos \left(\frac{V_{\mathrm{dc}}}{V_{A}}\right)\right\} .
\end{aligned}
$$

Equation (9) as well as the equation based on a vertical diode $^{14}$ indicate that the maximum frequency of the organic rectifier depends on the values of the required dc voltage and the provided ac supply voltage. We plot $f_{\max }$ vs the generated $V_{\mathrm{dc}}$ in Fig. 9 assuming the following values $V_{A}=20 \mathrm{~V}$, $V_{F}=3.5 \mathrm{~V}, \mu=0.15 \mathrm{~cm}^{2} / \mathrm{V} \mathrm{s}$, and $L_{D}=160 \mathrm{~nm}$ for a vertical diode and $\mathrm{V}_{A}=20 \mathrm{~V}, V_{T}=2.4 \mathrm{~V}, \mu=0.8 \mathrm{~cm}^{2} / \mathrm{V} \mathrm{s}, L=3 \mu \mathrm{m}$, 


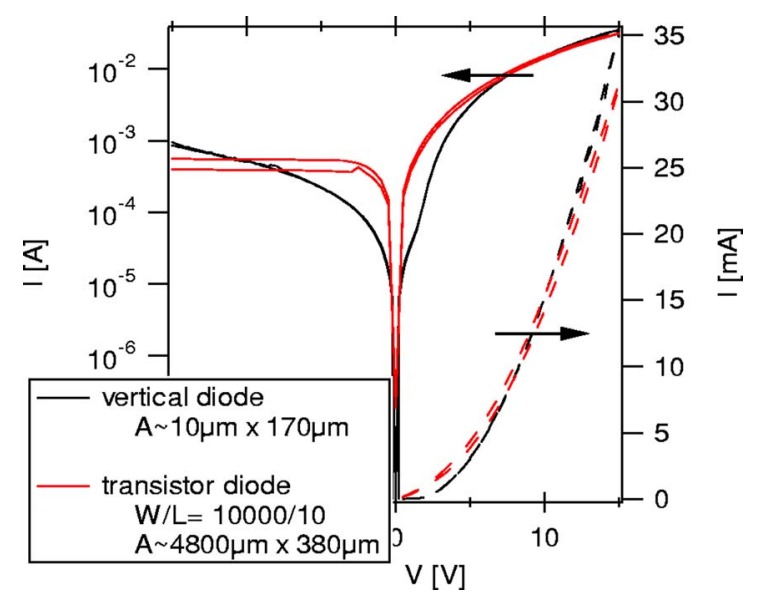

FIG. 10. (Color online) Current-voltage characteristics of the vertical pentacene diode $\left(10 \times 170 \mu \mathrm{m}^{2}\right)$ vs a transistor with shorted drain-gate $\left(W / L=10000 / 10 \rightarrow A \sim 4800 \times 380 \mu \mathrm{m}^{2}\right.$; if channel length is reduced with proportional scaling of width, the current-voltage characteristics remain unchanged but the area consumption decreases accordingly; $L=5 \mu \mathrm{m} \rightarrow A \sim 1600 \times 400 \mu \mathrm{m}^{2}$, and $L=3 \mu \mathrm{m} \rightarrow A \sim 800 \times 400 \mu \mathrm{m}^{2}$ ).

and $L_{\text {overlap }}=6 \mu \mathrm{m}$ for the transistor-based diode. It can be seen that with these parameters, frequencies up to 13.56 $\mathrm{MHz}$ are intrinsically possible. The maximum frequency possible is more than two orders of magnitude higher for the vertical diode. In the limit, a vertical diode may even allow to operate in the UHF frequency range. At $13.56 \mathrm{MHz}$ in the transistor diode there is already a significant loss of rectified dc voltage.

An additional problem which is not immediately obviously for the transistor diode is its high capacitance in comparison to the vertical diode. In Fig. 10, a vertical diode and a transistor-based diode with a similar $I-V$ curves can be seen. Depending on the channel length used, the transistor diode requires a several hundred times lager area as compared to the vertical diode, which automatically translates in a several hundred times lager capacitance of the transistor diode.

If we assume a load of $R_{L}=50 \mathrm{k} \Omega$ and an allowed ac fluctuation on top of the dc signal of $1 \%$ at a frequency of 13.56 MHz, from Eq. (2) we estimate that the minimum load capacitance is about $150 \mathrm{pF}$. Under the assumption that the capacitance is realized in the same layers as the circuit, i.e., using the gate dielectric of the transistor as capacitance dielectric, this translates in an area of approximately $A \sim 600 \times 600 \mu \mathrm{m}$.

Furthermore, the load capacitance needs to satisfy Eq. (3). In other words, the size of the load capacitance is also determined by the size of the diode capacitance, which is by itself determined by the required current that the diode needs to provide. If we solve Eq. (1) without substituting the load as done in Eqs. (2)-(4), we can directly get a frequency-independent value for the minimum size of the required diode by assuming a load of $50 \mathrm{k} \Omega$ and otherwise the same values as used for calculating Fig. 9. Under the condition that frequency limitations do not play a role, we need a vertical diode with an area of $A \sim 10 \times 10 \mu \mathrm{m}^{2} \quad\left(C_{\text {vertical diode }} \sim 16.6 \mathrm{fF}\right)$. The transistorbased diode requires a $W / L \sim 305$ translating into a capaci- tance of $C_{\text {transistor diode }} \sim 3.4 \mathrm{pF}$. The load capacitance determined by Eq. (3) would need to be at least $340 \mathrm{pF}$ translating in a minimum size of $900 \times 900 \mu \mathrm{m}^{2}$, even larger than the load capacitance required by Eq. (2). We conclude that not only frequency wise but also area wise the vertical diode structure offers much more performance than the transistorbased diode. High-frequency performance as well as area consumption are in the transistor based diode strongly dependent on the possible resolution and alignment accuracy of the process. By contrast, the challenge in getting sufficient performance in a vertical diode lays in the required high purity and low trap density of the organic semiconductor to achieve SCLC mobility in the same order of magnitude as compared to the field effect mobility. Resolution and alignment accuracy play a less significant role using a vertical diode.

In summary, we have compared a vertical Schottky diode and a transistor with shorted drain-gate regarding their performance in a rectifying circuit. The appealing property of a transistor based diode is its ease of integration with the logic gates. However, it has limitation concerning frequency and area consumption. Our experiments have shown that both structures can achieve sufficient rectification at 13.56 $\mathrm{MHz}$ to drive a circuit. However, the transistor-based diode has at this frequency a significantly reduced output voltage in comparison to the vertical diode. In addition, it has significantly higher area consumption for the diode as well as for the required load capacitance.

\section{ACKNOWLEDGMENT}

This work was partially supported by the Europeanfunded Integrated Project POLYAPPLY (IST No. 507143).

${ }^{1}$ B. K. Crone, A. Dodapalapur, R. Sarpeshkar, A. Gelperin, H. E. Katz, and Z. Bao, J. Appl. Phys. 91, 12 (2002).

${ }^{2}$ H. Klauk, M. Halik, U. Zschieschang, F. Eder, G. Schmid, and C. Dehm, Appl. Phys. Lett. 82, 23 (2003).

${ }^{3}$ P. F. Baude, D. A. Ender, M. A. Haase, T. W. Kelley, D. V. Muyres, and S. D. Theiss, Appl. Phys. Lett. 82, 22 (2003).

${ }^{4}$ T. W. Kelley, D. V. Muyres, P. F. Baude, T. P. Smith, and T. D. Jones, Mater. Res. Soc. Symp. Proc. 771, L6.5.1 (2003).

${ }^{5}$ K. Finkenzeller, RFID Handbook (Wiley, New York, 2002), Vol. 1, Chap. 5, p. 114, http://www.epcglobalinc.org/standards_technology/ specifications.html.

${ }^{6}$ D. de Leeuw, U.S. Patent No. WO99/30432 (17 June, 1999).

${ }^{7}$ C. Tanase, E. J. Meijer, P. W. M. Blom, and D. M. de Leeuw, Phys. Rev. Lett. 91, 216601 (2003).

${ }^{8}$ E. Cantatore, T. Geuns, A. Gruijthuijsen, G. Gelinck, S. Drews, and D. de Leeuw, ISSCC 2006, Digest of Technical Papers, 2006, San Francisco, Feb. 5-9, 2006, pp. 272-273.

${ }^{9}$ S. Karg, M. Meier, and W. Riess, J. Appl. Phys. 82, 1951 (1997).

${ }^{10} \mathrm{~T}$. Aernouts, W. Geens, J. Poortmans, J. Nijs, and R. Mertens, Synth. Met. 122, 153 (2001)

${ }^{11}$ L. S. Roman, M. Berggren, and O. Inganäs, Appl. Phys. Lett. 75, 22 (1999).

${ }^{12}$ W. Hu, B. Gompf, J. Pflaum, D. Schweitzer, and M. Dressel, Appl. Phys. Lett. 84, 23 (2004).

${ }^{13}$ W. Gao and A. Kahn, Org. Electron. 3, 53 (2002). Z. T. Zhu, J. T. Mason, R. Dieckmann, and G. G. Malliaras, Appl. Phys. Lett. 81, 24 (2002).

${ }^{14}$ S. Steudel, K. Myny, V. Arkhipov, C. Deibel, S. De Vusser, J. Genoe, and P. Heremans, Nat. Mater. 4, 597 (2005).

${ }^{15}$ S. De Vusser, S. Steudel, K. Myny, J. Genoe, and P. Heremans, Mater. Res. Soc. Symp. Proc. 870E, H1.4.1 (2005).

${ }^{16}$ Z. T. Zhu, J. T. Mason, R. Dieckmann, and G. G. Malliaras, Appl. Phys. Lett. 81, 24 (2002). 
${ }^{17}$ M. Pope and C. E. Swenberg, Electronic Processes in Organic Crystals and Polymers, 2nd ed. (Oxford University Press, New York, 1999), Vol. 1, Chap. VI, p. 663.

${ }^{18}$ M. A. Lampert and P. Mark, Current injection in Solids (Academic Press, New York, 1970).

${ }^{19}$ D. Knipp, R. A. Street, A. Volkel, and J. Ho, J. Appl. Phys. 93, 347 (2003).
${ }^{20}$ D. Knipp, R. A. Street, and A. R. Volkel, Appl. Phys. Lett. 82, 22 (2003).

${ }^{21}$ U. Wolf, V. I. Arkhipov, and H. Baessler, Phys. Rev. B 59, 11 (1995).

${ }^{22}$ L. Ma, J. Ouyang, and Y. Yang, Appl. Phys. Lett. 84, 23 (2004).

${ }^{23}$ H. Klauk, M. Halik, F. Eder, G. Schmid, and C. Dehm, IEDM 2004, Technical Digest, San Francisco, Dec. 13-15, 2004, pp. 369-372.

${ }^{24}$ S. M. Sze, Physics of Semiconductor Devices, 2nd ed. (Wiley, New York, 1981). 